\title{
ИНФУЗИОННАЯ ТЕРАПИЯ: РЕКОМЕНДАЦИИ НА ОСНОВЕ ЗДРАВОГО СМЫСЛА
}

\author{
(обзор британского гайдлайна“ по инфузионной терапии, \\ адаптированный У.А.Фесенко) \\ Харьковский национальный медицинский университет
}

* Intravenous fluid therapy in adults in hospital (National Institute for Health and Care ExcellenceClinical Guideline, UK. December, 2013) guidance.nice.org.uk/cg174

\section{Принципы назначения жидкостей}

Для того чтобы заложить основы безопасной и эффективной внутривенной (в/в) жидкостной терапии, необходимы знания в следующих областях:

- физиология водного баланса в норме;

· патофизиологические эффекты на водный баланс;

- клинические подходы для оценки потребности в жидкости;

свойства доступных в/в растворов.

\section{Физиология водного баланса в норме}

Когда примитивные морские одноклеточные организмы развиваются внугри многоклеточных организмов и перемещаются на берег, они переносят свое собственное «внутреннее море», или внеклеточную жидкость, в которой их клетки могут плавать в постоянном химическом окружении. Французский физиолог Клод Бернар назвал это «milieuinterieur», среда, в которой клетки сохраняют свой объем потребления энергии для выкачиванияионов натрия из клетки и закачивания ионов калия внутрь клетки, чтобы нейтрализовать негативные заряды белков и других ионов.

Водный баланс обычно подразумевает обмен воды между организмом и окружающей средой, то есть внешний баланс. Болезнь нарушает внутренний баланс между водными секторами организма, например, между внутрисосудистым и интерстициальным компонентами внеклеточного сектора, между внутриклеточным и внеклеточным секторами, между внеклеточным сектором, кишечником и другими внутренними пространствами.

Внутривенная жидкостная терапия зависит от понимания процессов, лежащих в основе физиологии и патофизиологии, и значимости не только наружного, но и внутреннего жидкостного баланса.

Нормальная анатомия и физиология водно-электролитного баланса

Доля воды составляет приблизительно $60 \%$ массы тела взрослого человека (около 40 лпри массе тела 70 кг). Процентное содержание воды ниже у людей с ожирением, так как жировая ткань содержит меньше воды, чем тощая ткань. Содержание воды у женщин меньше, чем у мужчин, поскольку у них относительно больше жировой ткани.

Общая вода организма функционально разделена на внеклеточный (около 20\% массы тела, или примерно14 л на 70 кг) и внутриклеточный (около 40\% массы тела, или примерно 28 л на 70 кг) водные сектора, разделенные клеточными мембранами с активными натриевыми насосами, которые обеспечивают преимущественное содержание ионов натрия во внеклеточном секторе. Клеткасодержит большие анионы, такие как белки и гликоген, которые не могут покинуть клетку, и поэтому притягивает внутрь ионы калия для поддержания электронейтральности (равновесие Гиббса-Доннана). Эти механизмы обеспечивают осмоляльность внеклеточной жидкости за счет натрия и его балансирующих

\footnotetext{
○ У.А.Фесенко, 2014
} 
анионов $\left(\mathrm{Cl}^{-}\right.$и $\left.\mathrm{HCO}_{3}^{-}\right)$. Калий играет соответствующую роль во внутриклеточной жидкости. Внеклеточный сектор в свою очередь разделяется на внутрисосудистый (объем циркулирующей крови (ОЦК)) и интерстициальный (внесосудистая вода, окружающая клетки) секторы. Внутрисосудистый сектор (объем крови - 5-7\% массы тела, приблизительно 4-5 л) имеет внутриклеточный компонент в виде эритроцитов (гематокрит $40-45 \%$ ) и лейкоцитов, а также внеклеточный компонент в виде плазмы (55-60\% ОЦК).

Внутрисосудистый и внесосудистый сектора разделены капиллярными мембранами, которые имеют микропоры. Внутрисосудистый объем зависит от онкотического (коллоиднорсмотического) давления плазмы, которую обеспечивают белки плазмы, притягивая воду в сосуды. Это давление в норме составляет около 3,4 кПа (26 мм рт.ст.), причем 75\% этого эффекта обеспечивают альбумины, 20\% гемоглобин и 5\% - глобулины. Концентрация альбумина в плазме - 35-52 г/л, общее его содержание в организме - около 270 г (120 г во внутрисосудистом секторе, 150 г в интерстициальной жидкости). 1 г альбумина связывает 18 мл воды.Таким образом, при нормальном содержании альбумин в плазме связывает 18 мл 120 г $=2,25$ л внутрисосудистой «плазменной» воды.

В норме микропоры капилляров обеспечивают медленный выход альбумина из сосудов (5\%/ч, 120 г/сут), который затем возвращается в циркуляторное русло в составе лимфы в таком же темпе, что способствует сохранению равновесия.

Под действием гидростатического давления в циркуляторном русле вода выталкивается из сосудов, а под действием онкотического давления белков, например, альбумина, притягивается в сосуды. Это поддерживает относительное постоянство объема плазмы в пропорции с внеклеточным сектором (эффект
Старлинга). Клинически важным является обмен жидкостью и электролитами между внеклеточным сектором и желудочнокишечным трактом (ЖКТ), включающий активную секрецию и реабсорбцию пищеварительных соков. В здоровом организме постоянно происходит обмен жидкости между разными секторами.Важные физиологические механизмы обеспечивают постоянное взаимодействие между ними, поддерживая внутренний жидкостный баланс.

\section{Жидкостный баланс}

Внешний жидкостный и электролитный баланс между организмом и окружающей средой поддерживается поступлением жидкостей и электролитов в организм извне и потерей их через почки, ЖКТ, кожу и легкие (неощутимые потери). В табл.1 приведены приблизительные цифры потери и поступления воды в сутки для взрослого человека. Эти цифры могут существенно изменяться в зависимости от климата, например, неощутимые потери воды и электролитов больше вжарком климате.

\section{Поступление}

В нормальных условиях большая часть воды в организм поступает с напитками. Вода также образуется в процессе оксидации, хоть и в небольшом количестве. Питье регулируется чувством жажды, которая появляется при отрицательном водном балансе вследствие

Таблица 1. Приблизительный суточный водный баланс у здорового взрослого, мл

\begin{tabular}{l|l|l|r}
\hline \multicolumn{1}{c|}{ Поступление } & \multicolumn{2}{c}{ Потери } \\
\hline $\begin{array}{l}\text { Вода, } \\
\text { содержащаяся в } \\
\text { напитках }\end{array}$ & 1200 & Моча & 1500 \\
\hline $\begin{array}{l}\text { Вода, } \\
\text { содержащаяся в } \\
\text { твердой пищи }\end{array}$ & 1000 & $\begin{array}{l}\text { Неощутимые } \\
\text { потери через } \\
\text { кожу и легкие }\end{array}$ & $500-1000$ \\
$\begin{array}{l}\text { Метаболическая } \\
\text { вода от оксидации }\end{array}$ & 300 & Кал & 100 \\
\hline
\end{tabular}


недостаточного поступления воды или повышенных ее потерь. Жажда также появляется при избыточном поступлении натрия, так как больше воды требуется для нормализации концентрации натрия во внеклеточной среде. Хоть у стариков чувство жажды притупляется, но оно обеспечивает соответствие поступления воды потребностям организма, поддерживая нулевой баланс и постоянную физиологическую осмоляльность на уровне 280-290 мосм/кг.

Средние суточные поступления:

вода - 25-35 мл/кг;

натрий - приблизительно 1 ммоль/кг;

калий - приблизительно 1 ммоль/кг.

Клод Бернар предложил термин "volume obligatoire» («обязательный объем») для описания минимального объема мочи, необходимого для выведения ненужных продуктов, например, мочевины, чтобы предотвратить их накопление в крови. Это понятие подразумевает, что если достаточное количество воды выпито или введено для покрытия неощутимых и других потерь и для потребностей почек, то нет преимущества в приеме большего объема воды. Избыточное поступление воды и электролитов может быть опасным при определенных условиях, так как оно может превысить способность почек выводить их избыток и поддерживать нормальный баланс. Избыток воды и натрия может вызвать отеки при условии расширения внеклеточного сектора как минимум на 2-3 л.

\section{Потери}

Неощутимые потери происходят все время без нашего ведома. В климате Великой Британии количество таких потерь составляет 0,5-1,0 л/сут, но в жарком климате, во время лихорадки, напряжения потери с потом могут составлять несколько литров с содержанием до 50 ммоль/л натрия.

В норме кишечник эффективно абсорбирует воду и электролиты.Потери воды со стулом составляют всего 100-150 мл/сут. Но при наличии болезней эти потери могут существенно увеличиться.

Почки являются главным органом регуляции водно-электролитного баланса и выведения ненужных излишков, а также регулятором секреции гормонов. Умеренные суточные колебания в поступлении воды и электролитов вызывают незначительные изменения осмоляльности плазмы, которая активирует осморецепторы. Это в свою очередь вызывает чувство жажды, регулирует экскрецию воды и натрия в почках. При патологических потерях объема крови и внеклеточной жидкости активируются объемные рецепторы, которые преобладают над осморецепторами. При больших изменениях объема плазмы почки менее способны к регуляции осмоляльности. Это имеет важное значение в некоторых клинических ситуациях.

\section{Регуляция водного обмена}

Осморецепторы, которые реагируют на изменение осмоляльности плазмы, расположены в гипоталамусе и передают сигналы гипофизу для повышения или уменьшения секреции вазопрессина (антидиуретического гормона (АДГ)). Разведение внеклеточной жидкости, включая плазму, поступлением воды с осмоляльностью ниже, чем у плазмы, вызывает снижение секреции АДГ, вследствие чего почки выделяют больше свободной воды, что приводит к разведению мочи. Дегидратация приводит к повышению концентрации внеклеточной жидкости, что увеличивает секрецию АДГ, вследствие чего почечные трубочки реабсорбируют больше воды, концентрируя мочу. В ответ на дегидратацию нормальная почка может повысить содержание мочевины в моче примерно в 100 раз. Таким образом, объем нормально продуцируемой мочевины в результате метаболизма белков может быть выведен всего в 500 мл мочи.

При дефиците воды соотношение уровня мочевины или осмоляльности в моче и плазме 
является показателем концентрационной способности почек. Возраст и болезни могут нарушать концентрационную способность почек, при этом больший объем мочи требуется для выведения такого же количества ненужных продуктов.

При повышенном катаболизме белков вследствие избыточного их поступления или ускорения катаболизма требуется больший объем мочи для очищения организма от повышенного количества мочевины.

Для оценки функции почекважно измерять объем и концентрацию (осмоляльность) мочи, а также учитывать имеющиеся у пациента метаболические изменения. Если плазменная концентрация мочевины и креатинина не изменена и не превышает норму, а объем мочи за последние 24 ч - достаточный, это означает, что поступление воды было адекватным и достигнут «достаточный объем» мочи.

Регуляция содержания ионов натрия $\left(\mathrm{Na}^{+}\right)$

Поскольку постоянство внеклеточной жидкости и ее соотношение с общей водой организма в большей степени зависят от осмотического эффекта натрия и его анионов, важно, что почки поддерживают баланс натрия в узком диапазоне. При дефиците натрия объем плазмы и внеклеточной воды уменьшается. При этом стимулируются рецепторы давления в циркуляторной системе, что приводит к повышению секреции ренина в почках. Это в свою очередь стимулирует секрецию надпочечниками альдостерона, который действует на почечные канальцы, вызывая реабсорбцию натрия и сберегая его в организме. При избыточном поступлении ионов натрия ренинальдостероновая система угнетается, позволяя увеличить выведение ионов натрия до восстановления баланса.

Механизм сохранения натрия очень эффективен, почки могут снижать концентрацию ионов натрия в моче до $<5$ ммоль/л. С другой стороны, даже в здоровом организме, избыток натрия медленно выводится. Возможно, потому, что физиологические системы человека развивались в жаркой, с низким содержанием натрия африканской среде, и до последней эпохи не подвергались избыточному поступлению ионов натрия.

Выделение предсердного натрий-уретического пептида в ответ на инфузию жидкостей, вероятно, связано больше с объемом (растяжение правого предсердия), чем с нагрузкой натрием. Механизм поддержания баланса натрия может нарушаться вследствие болезней, что приводит к дефициту натрия или чаще всего - к избыточной задержке натрия с развитием отеков и побочными клиническими эффектами.

\section{Регуляция содержания ионов калия $\left(\mathrm{K}^{+}\right)$}

Несмотря на то, что во внеклеточном секторедоля ионов калия небольшая, его концентрация должна поддерживаться в узком диапазоне (3,5-5,3 ммоль/л), чтобы предотвратить риск мышечной дисфункции или потенциально фатальных сердечных осложнений. Это достигается путем обмена ионов $\mathrm{K}^{+}$в трубочках почек на ионы $\mathrm{Na}^{+}$или $\mathrm{H}^{+}$, что позволяет увеличить или уменьшить выделение ионов $\mathrm{K}^{+}$. При дефиците ионов $\mathrm{K}^{+}$, реабсорбция ионов $\mathrm{H}^{+}$нарушается, что приводит к гипокалиемическому алкалозу и снижает способность почек выделять ионы натрия.

\section{Патофизиологические эффекты на водный баланс}

Болезнь и повреждения влияют на водноэлектролитный баланс и распределение жидкости несколькими путями:

- неспецифический метаболический ответ на стресс (особенно при тяжелой болезни и повреждениях);

изменение регуляции водно-электролитного баланса, напрямую связанное со специфической дисфункцией органа или систем органов, действием лекарств или 
внутривенными препаратами для лечения данных заболеваний;

- изменение регуляции водно-электролитного баланса вследствие резкого ограничения приема пищи или голодания.

Неспецифический ответ на болезнь или повреждение

В 1930-х годах Cuthbertson описал метаболические изменения, которые возникают в ответ на повреждение (включая операцию и сепсис), - повышение метаболизма и разрушение белков для покрытия потребностей заживления. Позже было показано, что эти изменения связаны с нейроэндокринными и цитокиновыми ответными реакциями и происходят в три фазы. Фаза упадка, или шоковая фаза, - короткая и смягчается ресусцитацией. Это позволяет перейти в катаболическую фазу, длительность и интенсивность которой зависят от тяжести повреждений и их осложнений. Если воспаление затихает, то наступает анаболическая фаза выздоровления или реабилитации. Параллельно с этими метаболическими изменениями происходят изменения физиологии водноэлектролитного баланса. В катаболической фазе повышается секреция АДГ, кортизола и альдостерона, особенно если имеет место снижение объема крови или внеклеточной жидкости. Это приводит к задержке ионов натрия и воды и потере ионов калия. Изначально нормальная или сниженная способность к выделению избытка ионов натрия и воды уменьшатся, что приводит к расширению внеклеточного пространства и отекам. Эти неспецифические реакции подразумевают, что олигурия является нормой в контексте серьезного заболевания или повреждения, следовательно, наличие олигурии не является показанием для повышенного введения ионов натрия, воды и плазморасширяющих препаратов, кроме случаев дефицита внутрисосудистого объема, например, при послеоперационном кровотечении. Задержка воды и ионов натрия после повреждения является естественным механизмом защиты внеклеточного и циркуляторного объема любой ценой. Это также объясняет тот факт, что у ослабленных пациентов легче вызвать избыток жидкостей и ионов натрия во время катаболической фазы. Поскольку вода задерживается так же, как и ионы натрия, гипонатриемиюлегко вызвать путем избыточного введения воды или гипотонических жидкостей. Следовательно, важно использовать кристаллоиды не только в соответствующем объеме, но и в соответствующей концентрации, особенно при наличии реакции на заболевание или повреждение, когда почки не в состоянии корригировать ошибки назначений, даже при отсутствии их значительного повреждения или другой почечной патологии.

Фаза реконвалесценции после серьезного заболевания или повреждения характеризуется не только возвращением к анаболизму, но и восстановлением способности почек выделять накопившийся избыток воды и ионов натрия. Эти периоды повреждения называют «фаза задержки натрия» и «фаза натрийуреза».

\section{Транскапиллярный выход альбумина}

Ответная реакция организма на тяжелое заболевание или повреждение включает также увеличение размеров пор в мембранах капилляров и повышение скорости транскапиллярного выхода альбумина до $300 \%$ по сравнению с нормой (от 5 до 13-15\%/ч). Следствием этого является уменьшение содержания альбумина в плазме, что приводит к снижению коллоидно-осмотического давления плазмы, уменьшению внутрисосудистого сектора, тогда как в интерстициальном секторе альбумин вызывает отеки. Этот феномен может длиться от нескольких часов до нескольких дней. За альбумином из сосудов в интерстициальное пространство перемеща- 
ются вода и натрий. Это приводит к еще более выраженному сужению внутрисосудистого сектора и расширению интерстициального. Поскольку возврат альбумина с лимфой в циркуляторное русло остается без изменений, конечным результатом данных сдвигов является гиповолемия с отеками.

\section{Калий}

Потери калия при тяжелом заболевании или повреждении связаны не только с повышенным его выделением в результате гиперсекреции кортизола и альдостерона, но и с катаболизмом белков и гликогена. При распаде белков внутри клеток с выходом продуктов распада (аминокислот) из клеток с ними снижается количество отрицательных зарядов внутри клеток. Ионы калия как катионы, которые регулируют содержание анионов, также выходят из клеток в интерстиций, а затем выделяются из организма.
В случае повышенного катаболизма и нарушения функции почек выход ионов калия из клеток может превысить способность почек к их выделению, что может привести к опасной гиперкалиемии.

В фазе выздоровления в клетках восстанавливается анаболизм белков и гликогена и клетки захватывают ионы калия, потому в эту фазу поступлениеих в организм должно быть повышено, чтобы избежать гипокалиемии, а также помочь почкам вывести излишек ионов натрия.

\section{Эффекты специфических органных и системных дисфункций}

Многие специфические клинические состояния, а также методы лечения, применяемые при них, могут влиять на регуляцию водно-электролитного баланса. Детальное обсуждение таких проблем невозможно в рамках данного гайдлайна. Некоторые примеры приведены в табл. 2.

Таблица 2. Проблемы, влияющие на в/в назначение жидкостей

\begin{tabular}{|c|c|}
\hline Орган/система & Соображения при назначении в/в жидкостей \\
\hline Сердечная дисфункция & $\begin{array}{l}\text { Повышенная опасность застойной сердечной недостаточности при передозировке жидкости и } \\
\text { натрия. Потенциальная опасность гипокалиемии, вызванной диуретиками и активацией ренин- } \\
\text { ангиотензин-альдостероновой системы, или гиперкалиемии от калий-сберегающих диуретиков. } \\
\text { Тяжелые сердечные пациенты могут также иметь сопутствующую почечную или печеночную } \\
\text { дисфункцию }\end{array}$ \\
\hline Почечные болезни & $\begin{array}{l}\text { Нарушение клиренса или повышенная потерякак жидкости, так и электролитов при острой и } \\
\text { хронической патологии почек. Нарушение обмена кальция и фоссрора при хронической } \\
\text { почечной недостаточности }\end{array}$ \\
\hline $\begin{array}{l}\text { Желудочно-кишечные } \\
\text { расстройства }\end{array}$ & $\begin{array}{l}\text { Повышенная потеря жидкости и электролитов. У пациентов с кишечной непроходимостью } \\
\text { огромное количество жидкости, богатой электролитами, секвестрируется в кишечнике }\end{array}$ \\
\hline Печеночные болезни & $\begin{array}{l}\text { Выраженные сдвиги водно-электролитного баланса с тенденцией к задержке воды и ионов } \\
\text { натрия вследствие комплексных патофизиологических изменений, включая } \\
\text { гиперальдостеронизм. У многих пациентов имеет место почечная недостаточность средней и } \\
\text { тяжелой степени - гепато-ренальный синдром }\end{array}$ \\
\hline Респираторные болезни & $\begin{array}{l}\text { Повышенные потери воды через легкие.Многие пациенты чувствительны к избытку жидкости. } \\
\text { Часто встречается синдром повышенной секреции АДГ (SIADH). «Легочное» сердце делает } \\
\text { пациентов уязвимыми к увеличению венозного объема, что иногда приводит к застойной } \\
\text { дисфункции печени }\end{array}$ \\
\hline Неврология & $\begin{array}{l}\text { Гипоталамо-гипофизарные болезни могут приводить к тяжелым нарушениям регуляции } \\
\text { водного баланса. Повышенную концентрацию натрия внутри вен иногда используют для } \\
\text { снижения внутричерепного давления }\end{array}$ \\
\hline Дерматология & $\begin{array}{l}\text { Ожоги и выраженные воспалительные заболевания кожи могут привести к повышенной потере } \\
\text { плазмы и жидкостей }\end{array}$ \\
\hline Эндокринология & $\begin{array}{l}\text { Эндокринные нарушения, включая сахарный диабет, Аддисонову болезнь, SIADH, могут } \\
\text { серьезно затруднить регуляцию водно-электролитного баланса }\end{array}$ \\
\hline
\end{tabular}


Диагностические критерии синдрома несоответствующей секреции АДГ (Syndrome of inappropriate ADH secretion (SIADH)):

- натрий в плазме $<130$ ммоль/л и осмоляльность плазмы $<275$ ммоль/кг;

- натрий в моче $>40$ ммоль/л и осмоляльность мочи $>100$ мосм/кг;

- отсутствие отеков и признаков гиповолемии;

- нормальная функция почек, щитовидной железы и надпочечников;

- пациент не получает диуретиков и слабительных препаратов.

Причины SIADH:

- онкопатология: мелкоклеточная карцинома бронхов, тимома, лимфома, саркома, мезотелиома, карцинома поджелудочной железы и двенадцатипалойкишки;

- патология внутригрудных органов: пневмония, туберкулез, эмпиема, астма, пневмоторакс, вентиляция с сопротивлением в конце выдоха (ПДКВ);

- неврологические заболевания: менингит, энцефалит, опухоль, абсцесс головного мозга, субарахноидальное кровоизлияние, синдром Гиена-Барре, острая перемежающаяся порфирия;

- лекарства: антидепрессанты, карбамазепин, цитостатики, «Экстази», опиоиды, окситоцин, фенотиазин, тиазиды;

- другие: послеоперационный период, надпочечниковая недостаточность, ВИЧ.

\section{Эффект голодания на водно- электролитный баланс}

Недоедание встречается часто у госпитализированных пациентов, являясь как причиной, так и следствием тяжелой болезни или повреждения. Недоедание может оказать неспецифическое влияние на водноэлектролитный статус, так как голодание сопровождается угнетением насосов клеточных мембран, вследствие чего поступление ионов натрия и воды в клетки увеличивается, а ионы калия, магния, кальция и фосфат выходят из клеток и выводятся почками. Таким образом, в организме голодающего имеет место избыток ионов натрия и воды и дефицит ионов калия, фосфата, магния и кальция. Эти изменения часто не выявляются, так как уровень этих ионов в плазме остается нормальным.

Проблема возникает с началом питания, даже если оно обеспечивается в виде глюкозы в составе инфузии. Поступление глюкозы в клетки вместе с инсулином, выделение которого она стимулирует, может восстановить функцию насосов мембран клеток с последующим захватом клетками ионов калия, фосфата, магния и кальция, что может привести к опасному снижению уровня этих ионов в плазме. В то же время ионы натрия и вода выходят из клеток в интерстиций и циркуляторное русло. Такое перераспределение в дополнение кв/в инфузиям может превысить резервные возможности сердца, обострить скрытые инфекции с повышением проницаемости капилляров. Это может привести к потенциально летальному избытку жидкости в организме и сердечной недостаточности. Данные проблемы известны под названием «рефидинг-синдром».Советы по его предупреждению и лечению можно найти в «NICE guideline on Nutrition Supportin adults».

\section{Клинический подход к оценке потребности в жидкостях}

Для поступления жидкостей и электролитов в организм всегда нужно использовать наиболее простой, безопасный и эффективный путь введения. Прием жидкостей через рот должен использоваться при любой возможности. Внутривенного введения жидкостей следует избегать у пациентов, которые в состоянии есть и пить. Возможность энтерального введения жидкостей через зонд следует рассматривать, если прием через рот невозможен, но функция ЖКТ позволяет такой путь введения. 
Біль, знеболювання і́ інтенсивна терапія но 302014

Любая инфузия - агрессивный метод лечения. Пероральному приему жидкостей препятствуют аппетит и жажда больного, кишечная и сосудистая стенка, которыев какой-то мере противостоят нашим ошибкам (от которых никто не застрахован). При введении жидкостей и лекарств в вену мы ставим организм перед фактом (и он должен бороться не только с болезнью, но иногда еще и с нашей агрессией).

\section{Свойства доступных в/в растворов}

Для в/в инфузионной терапии доступных растворов кристаллоидов, синтетических коллоидов и альбумина.

Существует теоретическое преимущество использования коллоидов вместо кристаллоидов при ресусцитации пациентов с гиповолемией, так как коллоиды дольше задерживаются в сосудистом русле. Кристаллоиды распространяются по всему внеклеточному сектору.Их применение ограничено из-за преходящего эффекта на объем плазмы. Однако такие соображения основаны на исследованиях, проведенных на эуволемических волонтерах, которые не имели ни сдвигов, связанных с заболеваниями, ни нарушений проницаемости капилляров. Исследования у гиповолемичных пациентов показали, что кристаллоиды у них вызывают намного более выраженный волемический эффект, чем у здоровых волонтеров. Преимущество коллоидов над кристаллоидами в случае необходимости увеличения внутрисосудистого сектора не доказаны.

Описание всех доступных в Великой Британии инфузионных растворов не входит в цели данного гайдлайна, но ознакомление с некоторымииз них, часто используемыми, поможет в выборе состава инфузий и понимании спорных вопросов при назначении в/в инфузий.
Состав часто используемых в Великой Британии кристаллоидов и коллоидов приведен в табл. 3 и 4.

Подробное описание доступных растворов с описанием их свойств и потенциальных «за» и «против» приведено ниже.

\section{Изотонический $\mathrm{NaCl}$}

$0,9 \% \mathrm{NaClc}$ добавлением калия и без него наиболее часто используемый раствор для в/в
Таблица 3. Электролитный состав часто используемых коллоидов

\begin{tabular}{|c|c|c|c|}
\hline Состав & Желатин & $\begin{array}{c}\text { Тетра- } \\
\text { гидрокси- } \\
\text { этилкрахмал }\end{array}$ & Альбумин \\
\hline $\begin{array}{l}\mathrm{Na}^{+}, \\
\text {ммоль/л }\end{array}$ & $145-154$ & $137-154$ & $100-160$ \\
\hline $\begin{array}{l}\mathrm{Cl}^{-}, \\
\text {ммоль/л }\end{array}$ & $103-145$ & $118-154$ & 128 \\
\hline $\mathrm{Na}: \mathrm{Cl}$ & $1,0-1,47: 1,0$ & $1,0-1,25: 1,0$ & - \\
\hline $\begin{array}{l}\mathrm{K}^{+} \\
\text {ммоль/л }\end{array}$ & $4,0-5,1$ & 4 & $\leq 2$ \\
\hline $\begin{array}{l}\text { Ацетат, } \\
\text { ммоль/л }\end{array}$ & 24 & $24-34$ & - \\
\hline $\begin{array}{l}\text { Малат, } \\
\text { ммоль/л }\end{array}$ & - & 5 & - \\
\hline $\begin{array}{l}\text { Октаноат, } \\
\text { ммоль/л }\end{array}$ & - & - & 6,4 \\
\hline $\begin{array}{l}\mathrm{Ca}^{2+}, \\
\text { ммоль/л }\end{array}$ & $1,0-6,5$ & 2,5 & - \\
\hline $\begin{array}{l}\mathrm{Mg}^{2+} \\
\text { ммоль/л }\end{array}$ & 1 & $1-1,5$ & - \\
\hline $\begin{array}{l}\text { Молекулярная } \\
\text { масса, Да }\end{array}$ & $30000-35000$ & 130000 & - \\
\hline $\begin{array}{l}\text { Степень } \\
\text { замещения }\end{array}$ & - & $0,4-0,42$ & - \\
\hline $\begin{array}{l}\text { Масса коллоида, } \\
\text { г/л }\end{array}$ & $\begin{array}{l}35(3,5 \%) \\
40(4,0 \%)\end{array}$ & $60(6,0 \%)$ & - \\
\hline $\mathrm{pH}$ & $7,1-7,7$ & $4,5-6,5$ & - \\
\hline $\begin{array}{l}\text { Теоретическая } \\
\text { осмолярность, } \\
\text { мосм/л }\end{array}$ & 274-301 & $286,5-308,0$ & 274 \\
\hline $\begin{array}{l}\text { Коллоидно- } \\
\text { осмотическое } \\
\text { давление при } \\
\text { температуре } 37^{\circ} \mathrm{C}\end{array}$ & $25,7-33,3$ & 36 & - \\
\hline
\end{tabular}


Таблица 4. Электролитный состав часто используемых кристаллоидов

\begin{tabular}{|c|c|c|c|c|c|c|c|c|c|c|}
\hline $\begin{array}{l}\frac{n}{\pi} \\
\frac{5}{0} \\
0 \\
ن\end{array}$ & 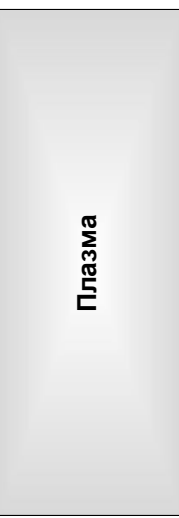 & 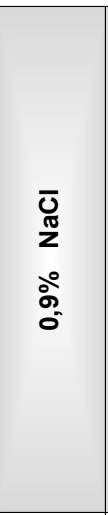 & 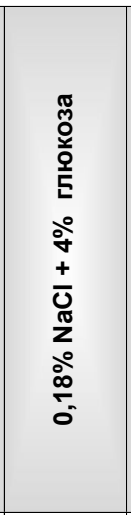 & 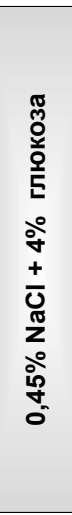 & 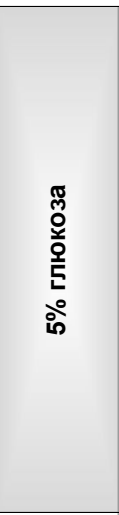 & 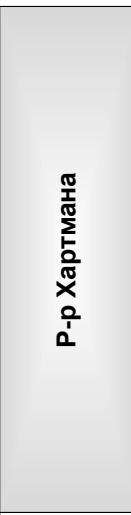 & 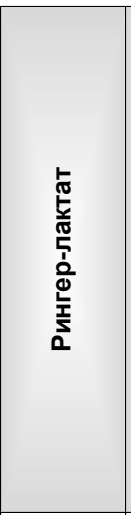 & 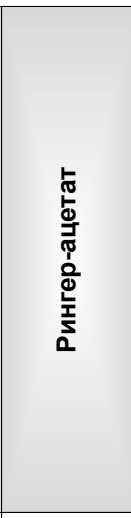 & 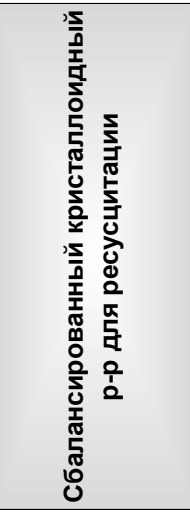 & 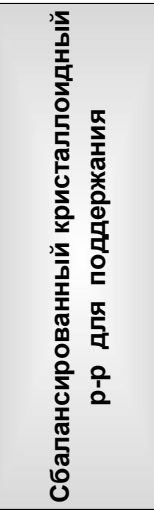 \\
\hline $\mathrm{Na}^{+}$,ммоль/л & $135-145$ & 154 & 31 & 77 & 0 & 131 & 130 & 130 & 140 & 40 \\
\hline $\mathrm{Cl}_{\text {,ммоль/л }}$ & $94-105$ & 154 & 31 & 77 & 0 & 111 & 109 & 112 & 98 & 40 \\
\hline $\mathrm{Na}: \mathrm{Cl}$ & $1,28-1,45: 1,0)$ & 1:01 & 1:01 & 1:01 & - & 1,18:1,00 & $1,19: 1,00$ & 1,16:1,00 & $1,43: 1,00$ & 1:01 \\
\hline $\mathrm{K}^{+}$,ммоль/л & $3,5-5,3$ & - & - & - & - & 5 & 4 & 5 & 5 & 13 \\
\hline $\begin{array}{l}\text { Бусрер, } \\
\text { ммоль/л }\end{array}$ & $\mathrm{HCO}_{3}^{-} 24-32$ & 0 & 0 & 0 & 0 & Лактат 29 & Лактат 28 & Ацетат 27 & $\begin{array}{l}\text { Ацетат - 27, } \\
\text { Глюконат - } 23\end{array}$ & Ацетат - 16 \\
\hline $\begin{array}{l}\mathrm{Ca}^{2+}, \\
\text { ммоль/л }\end{array}$ & $2,2-2,6$ & 0 & 0 & 0 & 0 & 2 & 1,4 & 1 & 0 & 0 \\
\hline $\begin{array}{l}\mathrm{Mg}^{2+}, \\
\text { ммоль/л }\end{array}$ & $0,8-1,2$ & 0 & 0 & 0 & 0 & 0 & 0 & 1 & 1,5 & 1,5 \\
\hline $\begin{array}{l}\text { Глюкоза, } \\
\text { ммоль/л }\end{array}$ & $3,5-5,5$ & 0 & $222(40$ г) & 0 & 278 (50г) & 0 & 0 & 0 & 0 & 0 \\
\hline $\mathrm{pH}$ & $7,35-7,45$ & $4,5-7,0$ & 4,5 & & $3,5-5,5$ & $5,0-7,0$ & $6,0-7,5$ & $6,0-8,0$ & $4,0-8,0$ & $4,5-7,0$ \\
\hline $\begin{array}{l}\text { Осмолярность, } \\
\text { мосм/л }\end{array}$ & 275-295 & 308 & 284 & & 278 & 278 & 273 & 276 & 295 & 389 \\
\hline
\end{tabular}

инфузий в Великой Британии, несмотря на возникшие вопросы о его соответствующем использовании. Как и все кристаллоиды 0,9\% $\mathrm{NaCl}$ распространяется по всему внеклеточному сектору, на объем плазмы его действие кратковременно по сравнению с коллоидами. Традиционно считалось, что инфузия $0,9 \% \mathrm{NaCl}$ увеличивает объем циркулирующей крови на 1/4-1/3 объема инфузии, остальная часть секвестрируется в интерстициальном пространстве. В действительности же, по причине, описанной выше, $0,9 \% \mathrm{NaCl}$ больше задерживается в сосудах у пациентов с гиповолемией и стрессом. Теоретически инфузия $0,9 \% \mathrm{NaCl}$ для расширения объема плазмы должна вызывать больше отеков, чем инфузия коллоидов. Но такое редко возникает на практике.

Меныший уровень задержки ненужных ионов натрия и воды является проблемой тогда, когда $0,9 \% \mathrm{NaCl}$ используют для рутинной поддержки. Суточная потребность в натрии 70-100 ммоль, так как 1 л 0,9\% $\mathrm{NaCl}$ содержит 154 ммоль натрия, таким образом, легко создать избыток ионов натрия, что может быть опасным при нарушении его экскреции на фоне тяжелой болезни или повреждения.

Другая причина для ограничения широкого применения $0,9 \% \mathrm{NaCl}$ вызвана тем, что содержание хлора в нем выше, чем в плазме, что приводит к гиперхлоремии. Это в свою очередь вызывает значительное снижение 
почечного кровотока и гломерулярной фильтрации, а также гиперхлоремический ацидоз, ацидоз в слизистой оболочке ЖКТ и илеуса (паралитическую кишечную непроходимость). Некоторые потери из ЖКТ и почечные потери способствуют выведению натрия и хлора, потому применение $0,9 \% \mathrm{NaCl}$ оправдано при повышенных потерях ионов натрия или при дефиците ионов натрия, хлора и воды вследствие предшествующих потерь. Важно помнить, что в патологических потерях содержится большое количествоионов калия, кальция и магния, поэтому сбалансированные кристаллоидные растворы имеют преимущество перед $0,9 \% \mathrm{NaCl}$ с добавлением калия.

Сбалансированные кристаллоидные pacmворы

Сбалансированные кристаллоидные растворы также распространяются по всему внеклеточному сектору и, следовательно, имеют такой же эффект, как и 0,9\% NaCl при использовании для увеличения объема плазмы. Они имеют теоретическое преимущество содержат несколько меньшее количество ионов натрия, значительно меньше хлорида, а также содержат ионы калия, кальция и магния. Использование сбалансированных кристаллоидных растворовдля ресусцитации или рутинной поддержки имеет преимущество перед применением 0,9\% NaCl. Растворы с более специализированной рецептурой для ресусцитации и поддержания, состав которых теоретически подходит для разных обстоятельств, возможно, будут более доступны в ближайшем будущем. Сбалансированные растворы с содержанием лактата или других буферов могут также иметь преимущества в случае тяжелого ацидоза, что часто встречается, когда требуется ресусцитация.

\section{Глюкоза и глюкозо-солевые растворы}

Растворы 5\% глюкозы и глюкозо-солевые растворы с содержанием калия или без него не используют для ресусцитации или восполнения значительных потерь электролитов. Эти растворы полезны для восполнения потерь свободной воды. Поскольку глюкоза метаболизируется и распространяется по всем водным секторам организма, она очень мало и в течение непродолжительного времени влияет на объем плазмы. Данные растворы полезны для коррекции простой дегидратации.Глюкозу с калием можно использовать для рутинного поддержания. Однако использование этих растворов связано с повышенным риском гипонатриемии, особенно при высокообъемных и быстрых инфузиях. Эти риски значительно выше у детей, у стариков, у пациентов, получающих диуретики, и при синдроме SIADH, который чаще встречается среди госпитализированных пациентов. Необходимо помнить, что в 5\% глюкозе очень малокалорий, поэтому данный раствор не способствует обеспечению энергией пациента.

\section{Синтетические коллоиды}

Синтетические коллоиды содержат некристаллизированные большие молекулы или ультрамикроскопические частицы, диспергированные в растворе, чаще - в кристаллоидах. Коллоидные частицы достаточно большие, чтобы удерживаться в циркуляторном русле и создавать онкотическое давление через капиллярную мембрану. Теоретически коллоиды, изоонкотичные с плазмой, должны расширять объем плазмы пропорционально введенному их объему. Но на практике увеличение объема плазмы происходит на 60$80 \%$. И этот показатель намного меньше у ослабленных пациентов с высоким уровнем транскапиллярных потерь. Настоящих преимуществ коллоидов над кристаллоидами при их использовании для расширения внутрисосудистого объема при ресусцитациии при проблемах с отечным перераспределением жидкости не доказаны. Более того, для некоторых препаратов из этой группы существуют доказательства того, что преимущества их 
нивелируются из-за потенциальных побочных эффектов со стороны почек и свертывающей системы.

Более старые препараты гидроксиэтилкрахмалов растворяются в $0,9 \% \mathrm{NaCl}$, тогда как новые препараты изготавливают на сбалансированных растворах, что делает их более «физиологичными». Все доступные на сегодня полусинтетические коллоиды содержат 140-154 ммоль натрия, что может вызвать положительный натриевый баланс у ослабленных пациентов, так же, как и использование 0,9\% $\mathrm{NaCl}$, хотя коллоиды содержат меньше хлоридов.

В Великой Британии синтетические коллоиды широко используют на догоспитальном этапе при транспортировке, в общих палатах, тогда как декстраны и высокомолекулярные (пента- и гексакрахмаллы) используют крайне редко или вообще не используют.

\section{Растворы альбумина}

Как иинфузия синтетических коллоидов, инфузия растворов альбумина имеет много теоретических преимуществ (например, лучшее расширение объема плазмы), хотя стоит альбумин очень дорого. Концентрированный (20-25\%), с низким содержанием натрия альбумин является ценным препаратом при проблемах перераспределения жидкости, особенно в случае отеков на фоне избытка воды и ионов натрия, что часто имеет место у пациентов после тяжелой болезни или повреждения, у которых снижен объем плазмы. Альбумин также применяют у некоторых пациентов с печеночной недостаточностью с асцитом, хотя описание его использования в данной ситуации не входит в рамки данного гайдлайна.

\section{ПРИНЦИПЫ И ПРОТОКОЛЫ ДЛЯ В/В ЖИДКОСТНОЙ ТЕРАПИИ}

Госпитализированные пациенты нуждаются во в/в введении жидкостей и электролитовпо одной или более из следующих причин (4R): Resuscitation - pесусцитация, Routinemaintenance - рутинное обеспечение физиологических потребностей, Replacement - восполнение патологических потерь, Redistribution - проблемы с внутренним перераспределением жидкости.

Жидкостная ресусичитациия

Ресусцитация - в/в введение жидкостей для немедленного обеспечения кровоснабжения жизненно важных органов после потери ОЦК вследствие кровотечения, плазмопотери или повышенного выведения жидкости и электролитов из организма, обычно через ЖКТ, или тяжелых внутренних потерь (например, вследствие перераспределения при сепсисе).

- У пациентов, нуждающихся во в/в жидкостной ресусцитации, используют кристаллоидные растворы с содержанием натрия 130-154 ммоль/л в виде болюса 500 мл в течение 15 мин.

Не используют тетракрахмаллы (tetrastarch) для жидкостной ресусцитации.

- Следует рассмотреть вопрос об использовании 4-5\% человеческого альбумина для жидкостной ресусцитации только у пациентов с тяжелым сепсисом.

\section{Рутинное поддержание}

Внутривенное введение жидкостей иногда необходимо пациентам, которые не могут обеспечить нормальные потребности в жидкости и электролитах путем приема их через рот или энтерально, но некоторое время выглядят вполне нормально, если не считать того, что водно-электролитный баланс нарушается и происходит перераспределение воды и электролитов. Однако, даже назначая в/в жидкости при более сложных случаях, нужно учитывать рутинную потребность, регулируя рутинное назначение с учетом жидкостных и электролитных проблем. Оценка потребностей для рутинного поддержания очень важна для всех 


пациентов при длительной жидкостной терапии.

- Если пациент нуждается вов/в жидкостной терапии только для рутинного поддержания, следует ограничить первоначальное назначение до 25-30 мл/кг/сут жидкости, которая должна содержать: приблизительно 1 ммоль/кг/сут калия, натрия и хлорида и 50-100 г/сут глюкозы для предупреждения голодного кетоза. Для большей информации табл. 5. Для пациентов с ожирением необходимо регулировать назначение жидкости в соответствии с их идеальной массой тела.

Таблица 5. Назначение в/в жидкости в зависимости от массы тела для рутинного поддержания В течение 24 ч

\begin{tabular}{|c|c|c|c|c|c|}
\hline $\begin{array}{c}\text { Масса } \\
\text { тела, кг }\end{array}$ & $\begin{array}{c}\text { Объем жидкости, } \\
\text { 25-30 мл/кг/сут }\end{array}$ & $\begin{array}{c}\text { Натрий, хлор, калий } \\
\text { (примерно } 1 \text { ммоль/кг/сут } \\
\text { каждого) }\end{array}$ & $\begin{array}{c}\text { Масса } \\
\text { тела, кг }\end{array}$ & $\begin{array}{l}\text { Объем жидкости, } \\
\text { 25-30 мл/кг/сут }\end{array}$ & $\begin{array}{c}\text { Натрий, хлор, калий, } \\
\text { (примерно } 1 \text { ммоль/кг/сут } \\
\text { каждого) }\end{array}$ \\
\hline 40 & $1000-1200$ & 40 & 71 & $1775-2130$ & 71 \\
\hline 41 & $1025-1230$ & 41 & 72 & $1800-2160$ & 72 \\
\hline 42 & $1050-1260$ & 42 & 73 & $1825-2190$ & 73 \\
\hline 43 & $1075-1290$ & 43 & 74 & $1850-2220$ & 74 \\
\hline 44 & $1100-1320$ & 44 & 75 & $1875-2250$ & 75 \\
\hline 45 & $1125-1350$ & 45 & 76 & $1900-2280$ & 76 \\
\hline 46 & $1150-1380$ & 46 & 77 & $1925-2310$ & 77 \\
\hline 47 & $1175-1410$ & 47 & 78 & $1950-2340$ & 78 \\
\hline 48 & $1200-1440$ & 48 & 79 & $1975-2370$ & 79 \\
\hline 49 & $1225-14-70$ & 49 & 80 & $2000-2400$ & 80 \\
\hline 50 & $1250-1500$ & 50 & 81 & $2025-2430$ & 81 \\
\hline 51 & $1275-1530$ & 51 & 82 & $2050-2460$ & 82 \\
\hline 52 & $1300-1560$ & 52 & 83 & $2075-2490$ & 83 \\
\hline 53 & $1325-1590$ & 53 & 84 & $2100-2520$ & 84 \\
\hline 54 & $1350-1620$ & 54 & 85 & $2125-2550$ & 85 \\
\hline 55 & $1375-1650$ & 55 & 86 & $2150-2580$ & 86 \\
\hline 56 & $1400-1680$ & 56 & 87 & $2175-2610$ & 87 \\
\hline 57 & $1425-1710$ & 57 & 88 & $2200-2640$ & 88 \\
\hline 58 & $1450-1740$ & 58 & 89 & $2225-2670$ & 89 \\
\hline 59 & $1475-1770$ & 59 & 90 & $2250-2700$ & 90 \\
\hline 60 & $1500-1800$ & 60 & 91 & $2275-2730$ & 91 \\
\hline 61 & $1525-1830$ & 61 & 92 & $2300-2760$ & 92 \\
\hline 62 & $1550-1860$ & 62 & 93 & $2325-2790$ & 93 \\
\hline 63 & $1575-1890$ & 63 & 94 & $2350-2820$ & 94 \\
\hline 64 & $1600-1920$ & 64 & 95 & $2375-2850$ & 95 \\
\hline 65 & $1625-1950$ & 65 & 96 & $2400-2880$ & 96 \\
\hline 66 & $1650-1980$ & 66 & 97 & 2425-2910 & 97 \\
\hline 67 & 1675-2010 & 67 & 98 & $2450-2940$ & 98 \\
\hline 68 & 1700-2040 & 68 & 99 & 2475-2970 & 99 \\
\hline 69 & 1725-2070 & 69 & 100 & $2500-3000$ & 100 \\
\hline 70 & 1750-2100 & 70 & $>100$ & 2500-3000 & 100 \\
\hline
\end{tabular}


Используют более низкие объемы на 1 кг массы тела (пациенты редко нуждаются в объемах более 3 л/сут) и просят помощи эксперта, если индекс массы тела пациента более $40 \mathrm{\kappa} / \mathrm{M}^{2}$.

- Необходимо помнить о назначении меньших объемов жидкости (например, 2025 мл/кг/сут) для следующих групп пациентов:

пожилые или ослабленные;

с почечными проблемами или сердечной недостаточностью;

с недоеданием и риском рефидингсиндрома.

- Назначая жидкости только для рутинного поддержания, следует рассмотреть использование 25-30 мл/кг/сут $0,18 \%$ хлорида натрия в 4\% глюкозе с добавлением калия 27 ммоль/л в первый день (возможны другие режимы для достижения этого). Назначение более 2,5 л/сут повышает риск гипонатриемии. Это исходное назначение, дальнейшие назначения должны основываться на результатах мониторинга.

- Следует рассмотреть возможность назначения в/в жидкостей для рутинного поддержания в течение дневных часов, чтобы улучшить сон и комфорт пациента.

\section{Восполнение}

У некоторых пациентов в/в жидкости используют для восполнения потер из внутрисосудистого и/или других водных секторов, хотя это неургентная ресусцитация, но необходима коррекция имеющегося водноэлектролитного дефицита или продолжающихся наружных потерь (обычно через ЖКТ или мочевыделительную систему, большие неощутимые потери могут быть вследствие лихорадки, пациенты с ожогами могут терять большие объемы жидкости, особенно плазмы). Иногда эти дефициты могут развиваться медленно с сопутствующей компенсаторной адаптацией перераспределения жидкости и электролитов в тканях, что необходимо учитывать при соответствующем восполнении потребностей (например,осторожное, медленное восполнение, чтобы снизить риск демиелинизации моста).Источники патологических потерь жидкости приведены в табл. 6.

\section{Перераспределение}

Дополнительно к наружнымпотерямводы и электролитов некоторые пациенты в больнице имеютвыраженное внутреннее перераспределение жидкости или ненормальное усвоение жидкостей. Этот тип проблем встречается преимущественно у тех пациентов с сепсисом, тяжелым заболеванием, после обширных хирургических операций, больших операций на сердце, с сопутствующей патологией печени или почек. У многих из этих пациентов развиваются отеки от натрия и избытка воды, у некоторых жидкость секвестрируется в ЖКТ или грудной/брюшной полостях.

- Необходимо регулировать в/в назначениев зависимости от физиологических потребностей, дефицита или избытка жидкости и электролитов, продолжающихся патологических потерь или ненормального перераспределения воды и электролитов.

Следует обратиться к эксперту, если пациент имеет комплексные проблемы с водно-электролитным балансом или тяжелые сопутствующие состояния, например:

- тяжелые отеки;

- тяжелый сепсис;

- гипо- или гипернатриемию;

- почечную, печеночную и/или сердечную недостаточность;

- послеоперационную задержку жидкости или перераспределение;

- недоедание или рефидинг-синдром. 
Таблица 6. Состав теряемой жидкости при патологических потерях

\begin{tabular}{|c|c|}
\hline Источник & Состав \\
\hline Лихорадка, дегидратация, гипервентиляция & Потеря чистой воды.Может вызвать гипернатриемию \\
\hline Рвота, промывание желудка & $\begin{array}{l}\mathrm{Na}^{+}-20-60 \text { ммоль/л, } \\
\mathrm{K}^{+}-14 \text { ммоль/л, } \\
\mathrm{Cl}^{-}-140 \text { ммоль/л, } \\
\mathrm{HCO}_{3}^{-}-60-80 \text { ммоль/л }\end{array}$ \\
\hline Желчь & $\begin{array}{l}\mathrm{Na}^{+}-145 \text { ммоль/л, } \mathrm{K}^{+}-5 \text { ммоль/л, } \\
\mathrm{Cl}^{-}-105 \text { ммоль/л, } \mathrm{HCO}_{3}^{-}-30 \text { ммоль/л }\end{array}$ \\
\hline Панкреатический сок & $\begin{array}{l}\mathrm{Na}^{+}-125-138 \text { ммоль/л, } \\
\mathrm{K}^{+}-8 \text { ммоль/л, } \mathrm{Cl}^{-}-56 \text { ммоль/л, } \\
\mathrm{HCO}_{3}^{-}-85 \text { ммоль/л }\end{array}$ \\
\hline Диарея, колостома & $\mathrm{Na}^{+}-30-140$ ммоль/л, $\mathrm{K}^{+}-30-70$ ммоль/л, $\mathrm{HCO}_{3}^{-}-20-80$ ммоль/л \\
\hline Еюностома или фистула & $\begin{array}{l}\mathrm{Na}^{+}-140 \text { ммоль/л, } \mathrm{K}^{+}-5 \text { ммоль/л, } \\
\mathrm{Cl}^{-}-135 \text { ммоль/л, } \mathrm{HCO}_{3}^{-}-8 \text { ммоль/л }\end{array}$ \\
\hline $\begin{array}{l}\text { Большие потери из тонкого кишечника через свежую } \\
\text { или высокую стому, фистулу }\end{array}$ & $\begin{array}{l}\mathrm{Na}^{+}-100-140 \text { ммоль/л, } \mathrm{K}^{+}-4-5 \text { ммоль/л, } \\
\mathrm{Cl}^{-}-75-125 \text { ммоль/л, } \mathrm{HCO}_{3}^{-}-0-30 \text { ммоль/л }\end{array}$ \\
\hline $\begin{array}{l}\text { Небольшие потери из тонкого кишечника через } \\
\text { сформировавшуюся стому или низкую фристулу }\end{array}$ & $\begin{array}{l}\mathrm{Na}^{+}-50-100 \text { ммоль/л, } \mathrm{K}^{+}-4-5 \text { ммоль/л, } \\
\mathrm{Cl}^{-}-25-75 \text { ммоль/л, } \mathrm{HCO}_{3}^{-}-0-30 \text { ммоль/л }\end{array}$ \\
\hline Полиурия & Необходимо определять уровень $\mathrm{Na}^{+}$и $\mathrm{K}^{+}$, объем мочи \\
\hline
\end{tabular}

Взаимосвязи между 4R (Resuscitation, Routinemaintenance, Replacement and Redistribution)

5-ая R - Reassessment-также является важным элементом ведения пациента

Решения об оптимальном количестве, составе и скорости введения в/в жидкостей должны основываться на внимательном обследовании пациента. Клинические прин-ципы, лежащие в основе таких решений, могут быть сформулированы в виде вопросов.

Нуждается ли мой пациент во в/в жидкостной ресусичтации?

Это первый вопрос, так как ургентная в/в жидкостная терапия является критическим элементом в лечении многих шоковых пациентов.

Может ли мой пациент обеспечить своипотребности в жидкости $и$ электролитах через рот или энтерально?

Необходимо избегать ненужных в/в инфузий жидкостей. Как только отпала необходимость в ней, она должна быть прекращена как можно раньше.

Каков настоящий водно-электролитный статус моего пациента?

Оценка должна проводиться с использованием всей доступной информации, включая анамнез, осмотр, клинический мониторинг (например, National Early Warning Score, водный баланс, масса тела) и лабораторные данные.

Каковы потребности моего пациента в воде и электролитах для рутинной поддержки?

В среднем взрослый нуждается в 25-30 мл/кг воды в сутки и около 1 ммоль/кг $\mathrm{Na}^{+}$и $\mathrm{K}^{+}$.

Имеет ли мой пациент дефиичт воды и электролитов или патологические продолжающиеся потери?

Все в/в назначения жидкостей должны обеспечить коррекцию дефицита воды/электролитов,восполнить продолжающиеся патоло- 


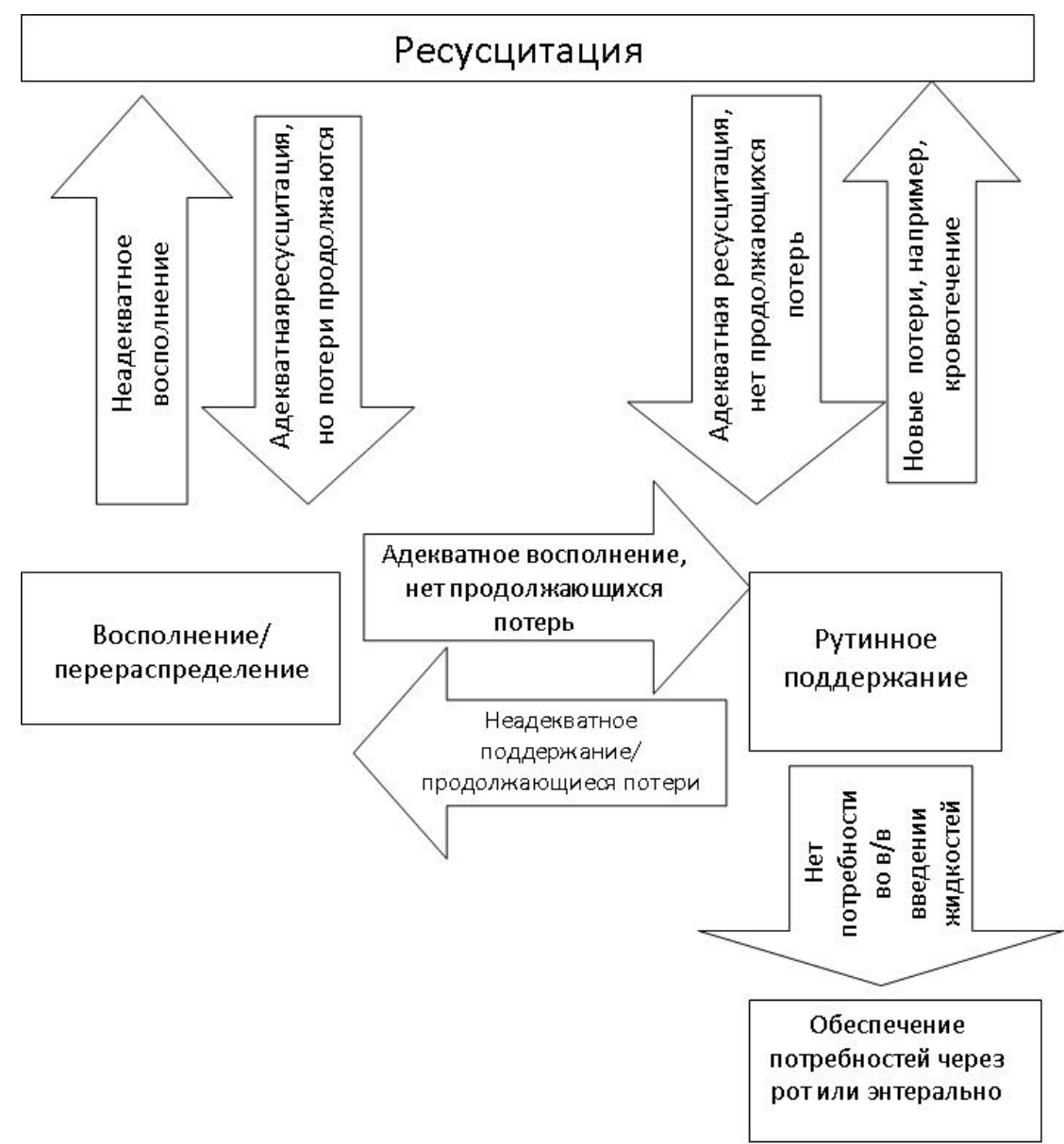

гические потери и обеспечить физиологические потребности.

Имеет ли мой пациент проблемы с внутренним перераспределением жидкости, или другие проблемы с регуляичией жидкостного баланса, связанные с основной патологией или с сопутствующими заболеваниями?

В/в назначение жидкостей должно проводиться с учетом как неспецифических ответных реакций на болезнь или повреждение, так и комплексных проблем с перераспределением жидкости, и изменений, связанных со специфическими органными и системными дисфункциями и/или голоданием.

Размышления над приведенными вопросами позволят определить общий объем жидкости и электролитный состав, которые должен полу- чить пациент, а также оптимальную скорость введения жидкостей. Скорость должна быть достаточно медленной, чтобы избежать перегрузки циркуляции, а также электролитных проблем, пока восстановится трансмембранное физиологическое равновесие между внеклеточным и внутриклеточным секторами.

Необходимо также выбрать оптимальный в/ в инфузионный раствор (или смесь растворов), принимая во внимание поступление жидкости/ электролитов в организм из всех источников (пища, напитки, энтеральное введение через зонд и в/в медикаменты). Кровь и в частности ее компоненты содержат большое количество электролитов, так же, как и в/в лекарства, особенно те, которые растворяются в большом объеме жидкостей и вводятся несколько раз в сутки. Пациенты, получающие искусственное 
парентеральное или энтеральное питание, обычно получают адекватное количество жидкости и электролитов в составе этих смесей, чтобы обеспечить рутинные потребности. Назначение дополнительной в/в инфузионной терапии у таких пациентов является распространенной ошибкой.

План в/в жидкостной терапии должен ежедневно оценивать эксперт,план для пациентов, находящихся на длительной жидкостной терапии, у которых состояние стабильное, может быть оценен реже.

Пациенты могут участвовать в составлении их жидкостного баланса. Если пациент нуждается вов/в жидкостях, необходимо объяснить ему решение врача, обсудить симптомы и признаки, за которыми он должен следить для нормализации его жидкостного баланса. По возможности или по просьбе пациента проводят письменное информирование, и привлекают семью пациента и ухаживающих.

\section{ОЦЕНКА И МОНИТОРИНГ}

\section{Исходная оценка}

- Необходимо определить наличие гиповолемии у пациента. Признаки того, что пациент может нуждаться в ургентной жидкостной ресусцитации:

- систолическое артериальное давление ниже 100 мм рт.ст.;
- частота сердечных сокращений более 90 уд./мин;

- $\quad$ время заполнения капилляров более 2 c

- или холодные на ощупь конечности;

- частота дыхания более 20 в 1 мин;

- оценка по Национальной шкале раннего реагирования (табл. 7) $\geq 5$.

Прикроватный тест на определение волемического статуса: пассивное поднятие ног свидетельствует о реакции гемодинамики на увеличение объема венозного возврата. Тест лучше проводить с исходной позицией пациента в полулежачем положении, приподнимая кровать на $45^{\circ}$. Альтернативный метод: у пациента, лежащего ровно на спине, пассивно поднимают обе ноги вместе более чем на $45^{\circ}$. Если в течение 30-90 с у пациента улучшаются гемодинамические параметры, то это свидетельствует о потребности восполнения объема. Если состояние пациента ухудшается, появляется одышка, то это свидетельствует об избытке жидкости у пациента.

· Необходимо определить вероятные потребности пациента в жидкости и электролитах поданным анамнеза, клинических данным, сведениям о получаемых лекарствах, данным клинического мониторинга и результатам лабораторного исследования:

из анамнеза: предшествовавшее ограничение приема жидкости, жажда, объем и

Таблица 7. Национальная шкала раннего реагирования (National Early Warning Score (NEWS))

\begin{tabular}{|c|c|c|c|c|c|c|c|}
\hline Параметры & 3 & 2 & 1 & 0 & 1 & 2 & 3 \\
\hline Частота дыхания в минуту & & $<8$ & $8-11$ & $12-20$ & $21-25$ & $26-30$ & $>30$ \\
\hline $\mathrm{SaO}_{2}, \%$ & $<85$ & $86-89$ & $90-94$ & $>95$ & & & \\
\hline ЧСС в минуту & & $<40$ & $41-50$ & $51-100$ & $101-110$ & $111-130$ & $>130$ \\
\hline АД & $<70$ & $71-80$ & $81-100$ & $101-179$ & 180-199 & $200-220$ & $>220$ \\
\hline Температура тела, ${ }^{\circ} \mathrm{C}$ & & $<35$ & $35,1-36,5$ & $36,6-37,4$ & $>37,5$ & & \\
\hline Неврологический статус & & & Возбужден & Тревожный & $\begin{array}{l}\text { Реагирует на } \\
\text { голос }\end{array}$ & $\begin{array}{l}\text { Реагирует на } \\
\text { боль }\end{array}$ & $\begin{array}{l}\text { Не } \\
\text { реагирует }\end{array}$ \\
\hline
\end{tabular}

Оценка $\geq 3$ баллов означает критическое состояниеи требует немедленного реагирования. Оценка не отражает полностью состояние пациента, но является руководством к действию 
состав патологических потерь (см. таблицу патологических потерь жидкости), любое сопутствующее заболевание, включая недоедание и риск рефидинг-синдрома; клиническое обследование предусматривает оценку жидкостного статуса пациента, включая частоту пульса, АД, время заполнения капилляров, наполнение шейных вен, наличие отеков в легких или периферических отеков, постуральной гипотензии; клинический мониторинг должен предусматривать оценку настоящего статуса и следующих параметров в динамике: оценку по NEWS, жидкостный баланс, масса тела; лабораторные исследования должны включать оценку настоящего статуса, клинический анализ кровии изучение в динамике содержания мочевины, креатинина и электролитов.

\section{Переоценка}

- Если пациент получает в/в жидкости для ресусцитации, следует переоценить пациента по схеме ABCDE (Airway - дыхательные пути, Breathing - дыхание, Circulation кровообращение, Disability - сознание, Exposure - наружные симптомы), мониторировать его частоту дыхания, пульса, АД, перфузию продолжительно, определить уровень лактата в венозной крови, $\mathrm{pH}$ и ВЕ в артериальной крови в соответствии с рекомендациями по продвинутой поддержке жизни (Advanced Life Support (ALS)), Resuscitation Council (UK), 2011).

- Bсе пациенты, получающие длительную в/в жидкостную терапию, нуждаются в регулярном мониторинге, который долженвключать по меньшей мере ежедневную переоценку жидкостного статуса, лабораторных данных (мочевина, креатинин и электролиты) и расчет водного баланса, взвешивание дважды в неделю. Следует помнить, что:

- пациенты, получающие в/в жидкостную терапию с целью восполнения и коррекции перераспределения, могут нуждаться в более частоммониторировании;

- дополнительный мониторинг уровня натрия в моче может быть полезным у пациентов с большой потерей жидкости из ЖКТ. Сниженная экскреция натрия с мочой (менее 30 ммоль/л) может свидетельствовать об истощении запасов натрия в организме, несмотря на нормальный уровень натрия в плазме. Уровень натрия в моче также может помочь в определении причины гипонатриемии и достижении отрицательного натриевого баланса у пациентов с отеками. Однако ценность определения уровня натрия в моче может ввести в заблуждение при наличии почечной недостаточности или терапии диуретиками; - у пациентов, которые находятся на длительной жидкостной терапии, состояние которых стабильное, можно выполнять мониторинг реже, хотя решение об этом должно быть обосновано в плане их жидкостной терапии.

Если пациенты получали в/в жидкости с содержанием хлорида более 120 ммоль/л (например, 0,9\% NaCl), то необходимо мониторировать плазменную концентрацию хлора ежедневно. Если у пациента развивается гиперхлоремия или ацидемия, то следует пересмотреть назначение жидкостей в/в и оценить его кислотно-основной статус. Необходимо рассмотреть возможность менее частого мониторинга для стабильных пациентов.

Про случаи ненадлежащего использования жидкостей (табл. 8) необходимо доложить как о стандартном критическом инциденте для улучшения тренинга и практики.

Если пациента переводят в другое отделение, то при поступлении в новое отделение следует переоценить его жидкостный статус и план жидкостной терапии. 


\section{Компетентность персонала в назначении жидкостной терапии}

Решение вопроса об оптимальных объемах, составах и скорости введения в/в жидкостей является сложной проблемой, но, несмотря на это, его часто поручают общим палатным врачам в больнице, часто - молодым врачам и сестрам, которые не имеют достаточного опыта и компетенции. Очевидно, что ненадлежащее использование жидкостей - распространенное

\section{Біль, знеболювання і́ інтенсивна терапія на з. 2.014}

явление, особенно в общих палатах, с потенциальными побочными результатами, включая повышение заболеваемости и смертности, удлинение сроков лечения в больнице и повышение стоимости лечения.

Необходимо разработать понятные рекомендации по назначению в/в жидкостей, подходящие для общих отделений. В большинстве рандомизированных контролируемых исследований в/в жидкостной терапии изучали узкие

Таблица 8. Последствия ненадлежащего проведения жидкостной терапии, о которых следует доложить как о критическом инциденте

\begin{tabular}{|c|c|c|}
\hline $\begin{array}{c}\text { Последствия } \\
\text { ненадлежащего } \\
\text { проведения } \\
\text { жидкостной терапии }\end{array}$ & Признаки идентификации & Временные рамки идентификации \\
\hline Гиповолемия & $\begin{array}{l}\text { Потребности пациента в жидкости не обеспечиваются } \\
\text { поступлением через рот, энтерально или в/в и имеются: } \\
\text { О признаки дегидратации при клиническом обследовании; } \\
\text { О снижение диуреза или концентрированная моча; } \\
\text { б биохимические данные, такие как увеличение на более чем } \\
50 \% \text { содержания мочевины или креатинина, без других } \\
\text { очевидных причин }\end{array}$ & $\begin{array}{l}\text { До начала и во время проведения в/в } \\
\text { жидкостной терапии }\end{array}$ \\
\hline $\begin{array}{l}\text { Отек легких (одышка во } \\
\text { время инфузии) }\end{array}$ & $\begin{array}{l}\text { Нет других очевидных причин (например, пневмония, } \\
\text { эмболия легочная или астма) } \\
\text { Признаки отека легких при клиническом обследовании } \\
\text { Признаки отека легких на рентгенограмме }\end{array}$ & $\begin{array}{l}\text { Во время проведения и в течение } \\
6 \text { ч после отмены в/в жидкостной } \\
\text { терапии }\end{array}$ \\
\hline Гипонатриемия & $\begin{array}{l}\text { Плазменный уровень натрия ниже } 130 \text { ммоль/л } \\
\text { Нет других очевидных причин гипонатриемии }\end{array}$ & $\begin{array}{l}\text { Во время проведения и в течение } \\
24 \text { ч после отмены в/в жидкостной } \\
\text { терапии }\end{array}$ \\
\hline Гипернатриемия & $\begin{array}{l}\text { Плазменный уровень натрия } \geq 155 \text { ммоль/л } \\
\text { Исходный уровень натрия нормальный или низкий } \\
\text { В/в инфузия включала 0,9 \% NaCl } \\
\text { Нет других очевидных причин гипернатриемии }\end{array}$ & $\begin{array}{l}\text { Во время проведения и в течение } \\
24 \text { ч после отмены в/в жидкостной } \\
\text { терапии }\end{array}$ \\
\hline Периферические отеки & $\begin{array}{l}\text { Ямочные отеки на конечностях и/или в пояснично- } \\
\text { крестцовой области } \\
\text { Нет других очевидных причин (например, нефротический } \\
\text { синдром или сердечная недостаточность). }\end{array}$ & $\begin{array}{l}\text { Во время проведения и в течение } \\
24 \text { ч после отмены в/в жидкостной } \\
\text { терапии }\end{array}$ \\
\hline Гиперкалиемия & $\begin{array}{l}\text { Плазменный уровень калия выше 5,5 ммоль/л } \\
\text { Нет других очевидных причин }\end{array}$ & $\begin{array}{l}\text { Во время проведения и в течении } \\
24 \text { часов после отмены в/в жидкостной } \\
\text { терапии. }\end{array}$ \\
\hline Гипокалиемия & $\begin{array}{l}\text { Плазменный уровень калия ниже 3,0 ммоль/л, очевидно, } \\
\text { вследствие инфузии растворов с низким содержанием } \\
\text { калия } \\
\text { Нет других очевидных причин (например, прием калий- } \\
\text { выводящих диуретиков, рефидинг-синдром) }\end{array}$ & $\begin{array}{l}\text { Во время проведения и в течение } \\
24 \text { ч после отмены в/в жидкостной } \\
\text { терапии. }\end{array}$ \\
\hline
\end{tabular}


клинические вопросы в отделениях интенсивной терапии или интраоперационных условиях. Большинство рекомендаций для общего пользования должны основываться на первичных принципах. Все сотрудники, причастные к назначению и проведению в/в жидкостной терапии, должны понимать эти принципы для того чтобы безопасно и эффективно проводить в/в жидкостную терапию.

\section{Тренинг и обучение}

- Госпитали должны следить за тем, чтобы все сотрудники,причастные к назначению и проведениюв/в жидкостной терапии, были обучены принципам, изложенным в данном гайдлайнсе, а также формально проэкзаменованы и переэкзаменованы через определенные интервалы времени для демонстрации компетенции в:

- понимании физиологии баланса жидкости и электролитов у здоровых и больных пациентов;

- оценке потребностей пациента в жидкости и электролитах (5R: Resuscitation, Routine maintenance, Replacement, Redistribution and Reassessment);

- оценке рисков, пользы и угроз в/в жидкостной терапии;

- назначении и использовании в/в жидкостей;

- мониторировании реакции пациента;

- оценке и документации изменений, а также правильных действиях при необходимости.

- Сотрудники здравоохранения должны проходить тренинг и обучение и быть компетентными в распознавании, оценке и предупреждении последствий ненадлежащего проведения в/в жидкостной терапии, включая:
- отек легких;

- периферические отеки;

- снижение объема и шок.

- Госпитали должны иметь руководителя по в/в жидкостной терапии, ответственного за тренинг, клиническое управление, аудит и оценку в/в назначения жидкостей и результаты у пациентов.

\section{Ключевые рекомендации для исследований:}

1. Какова инцидентность осложнений во время и после в/в жидкостной терапии?

2. Есть ли преимущества сбалансированных растворов по сравнению с 0,9\% $\mathrm{NaCl}$ для жидкостной ресусцитации у пациентов с острым гиповолемическим шоком?

3. Есть ли преимущества сбалансированных кристаллоидных растворов по сравнению с комбинацией их с суспензированным желатином для жидкостной ресусцитации у пациентов с острым гиповолемическим шоком?

4. Может ли более высокое содержание натрия в составе в/в жидкостной терапии снизить риск развития гипонатриемии, снижения объема, без повышения риска перегрузки объемом у госпитализированных взрослых пациентов?

5. Может ли выполняться введение в больницах систем, которые обеспечивают следующие условия:

- все сотрудники, причастные к назначению и проведениюв/в жидкостной терапии, имеют соответствующие навыки по назначениюинфузий;

- все осложнения в/в жидкостной терапии докладываются;

- привести к снижению количества осложнений в/в жидкостной терапии и связанных с ними затрат здравохранения? 